\title{
Multiparametric MRI Approach to Prostate Cancer with a Pictorial Essay on PI-RADS
}

\author{
Palak B. Popat ${ }^{1}$ Sharad Maheshwari \\ ${ }^{1}$ Department of Radiodiagnosis, Tata Memorial Hospital, Mumbai, \\ Maharashtra, India \\ 2Department of Radiodiagnosis, Kokilaben Dhirubhai Ambani \\ Hospital, Andheri, Mumbai, Maharashtra, India
}

Nilesh P. Sable ${ }^{1}$

Meenakshi Thakur ${ }^{1}$

Aparna Katdare ${ }^{1}$

\begin{abstract}
Address for correspondence Palak B. Popat, DNB, Department of Radiodiagnosis, Tata Memorial Hospital, Dr E Borges Road, Parel, Mumbai 400012, Maharashtra, India (e-mail: dr.palakp@gmail.com).
\end{abstract}

\begin{abstract}
Keywords

- multiparametric magnetic resonance imaging

- PI-RADS

- prostate cancer

The biology of prostate cancer is indolent, and incidence does not reflect mortality. This has led to reframed screening guidelines pivoting around serum prostate-specific antigen (PSA) and conceptualizing clinically significant prostate cancer (CSC), triaging active surveillance and intervention. A resultant paradigm shift in magnetic resonance imaging (MRI) from diagnosing cancer to focusing on detecting CSC led to the establishment of PI-RADS v2 (prostate imaging reporting and data systems, version 2). In this article, we present an approach to analyzing suspicious prostate lesions on multiparametric MRI (mp-MRI) and assigning them a PI-RADS assessment score based on the current version 2 for standardized reporting, strengthening diagnostic accuracy, and improving clinical acceptance. We also present pitfalls and challenges that a radiologist should be aware of, for increasing diagnostic accuracy.
\end{abstract}

\section{Introduction}

Prostate cancer is an insidiously growing malignancy, which may never clinically manifest in the lifetime of an individual. It is the second most common cancer in men. Its prevalence increases with age, and the histologic prevalence is as high as $70 \%$ in males aged $\geq 80$ years. ${ }^{1}$ Approximately 1.1 million men were estimated worldwide to be diagnosed with prostate cancer in 2012, accounting for $15 \%$ of the cancers diagnosed in men. As per the Surveillance, Epidemiology and End Results Program (SEER) data reflecting U.S. population statistics, the incidence of prostate cancer per lakh of population has dropped from 234.3 in 1992 to 99 in 2015, whereas mortality dropped from 39.2 to 18.9 . The 5 -year disease-free survival was 98.2\% from 2008 to $2014 .{ }^{2}$ Higher incidence and mortality rates prevail in developed regions, such as Europe and the United States, but the mortality to incidence ratio is approximately 2.5 times higher in less developed countries. ${ }^{3}$ In India, the incidence of detection of prostate cancer is rising significantly, being the second and third most common cancer in some metropolitan cities. ${ }^{4}$ It is most common in the age group $>65$ years. The estimated age-adjusted incidence rates (AARs) of prostate cancer

received in India as a whole was 3.7 per lakh persons during the year 2008. There is, however, wide heterogeneity in reporting cases and incidence across the country. ${ }^{5}$ Detection is expected to further rise with increasing life expectancy, growing awareness of the disease, urban migration, improvement in health services, and increasing utility of PSA testing.

Imaging aids in the diagnosis and management of prostate cancer. Among the imaging modalities, after PSA, mp-MRI with a PI-RADS v2 (prostate imaging reporting and data systems, version 2) assessment score has established its role in the early detection of CSC, staging and management of treatment, thereby stratifying patients for active surveillance versus intervention. There is unfortunately a vast heterogeneity in imaging acquisition techniques and reporting standards. Thus, improved standardization is the need for better clinical acceptance.

\section{Current Status of Multiparametric MRI}

MRI prostate has evolved from routine pelvic imaging to dedicated mp-MRI. Mp-MRI constitutes a series of magnetic resonance sequences of the prostate combining anatomical and functional imaging to detect and characterize prostate 
lesions, best analyzed on the prebiopsy gland, aiming to detect CSC. There is no universally accepted definition for CSC. However, for the purpose of standardizing MRI interpretation and pathology correlation, the European Society of Uroradiology (ESUR) has defined CSC as (1) histology with Gleason's score of $\geq 7$ (including both $3+4$ and $4+3$ ), (2) volume $\geq 0.5 \mathrm{cc}$, and/or (3) extraprostatic extension. ${ }^{6,7}$ Gleason's score, a pathologic scoring system is the sum of two histologic patterns seen in a specimen. On biopsy sample, the least score assigned is $6(3+3)$. Higher scores reflect higher grade of tumor and poorer prognosis. ${ }^{8,9}$ The tumor volume, Gleason's score, architecture, and location influence tumor detection on MRI. MRI is less sensitive in detecting the less aggressive Gleason's score $6(3+3)$ tumors, with tumor detection improving with increasing grade of tumor. Hence, the true strength of MRI is in the detection of aggressive (clinically significant) tumors. ${ }^{10}$ Prebiopsy MRI in a setting of elevated PSA levels leads to an equal or mildly increased detection rate of CSC, reduces the number of biopsies, and lowers the rate of diagnosis of clinically insignificant cancers. ${ }^{11}$ It localizes suspicious lesions for prostate biopsy, using fusion MRI-ultrasound-guided biopsy. MRI is particularly helpful in precise locoregional staging and assessment of lesion size, site, extraprostatic extension (EPE), and involvement of adjacent structures. Mp-MRI, especially diffusion-weighted imaging (DWI) and DCE, plays a role in selection of patients for surveillance, imaging the posttreatment prostate, and detection of recurrence. ${ }^{12}$ Mp-MRI offers a higher negative predictive value than systematic biopsy as was shown in the prostate MRI Study (PROMIS) trial, (89\% vs. 74\%). ${ }^{11}$ ACR (American College of Radiology) appropriateness criteria rate MRI as "usually appropriate" with a rating scale of 7 for clinically suspected prostate cancer in biopsy-naïve patients and a higher rating scale of 8 for clinically suspected prostate cancer with prior negative transrectal ultrasound-guided biopsy; for surveillance in low-risk cancer; for staging and/ or surveillance in intermediate-risk prostate cancer; and for staging in high-risk cancer. ${ }^{13}$ NCCN (National Comprehensive Cancer Network) guidelines recommend MRI in clinically suspected cancer when life expectancy is $>5$ years-for patients with rising PSA in prior negative biopsy, for active surveillance, and for PSA persistence and recurrence. ${ }^{14,15}$ - Table 1 summarizes the indications of mp-MRI in different clinical scenarios (- Table $\mathbf{1}$ ).

\section{Anatomy of the Prostate on mp-MRI}

Cancers of the prostate often are multifocal, with 80 to $85 \%$ arising from the peripheral zone (PZ), 10 to $15 \%$ arising from the transition zone (TZ), and 5 to $10 \%$ arising from the central zone (CZ). The zonal model of anatomy was originally described by McNeal. ${ }^{16}$ The prostate gland comprises of an outer PZ that is normally $\mathrm{T} 2$ bright and an inner $\mathrm{TZ}$ that is usually smaller with homogenous T2 intermediate signal intensity (SI) in young men and larger with heterogeneous SI in the middle age and elderly due to age-related changes of benign prostatic hyperplasia (BPH). CZ is the posterosuperiorly compressed
Table 1 Indications of mp-MRI prostate

\begin{tabular}{|l|l|}
\hline \multicolumn{2}{|l|}{ Indications for mp-MRI of the Prostate } \\
\hline $\begin{array}{l}\text { For treatment-naïve } \\
\text { patients }\end{array}$ & $\begin{array}{l}\text { Detecting CSC by PI-RADS v2 } \\
\text { assessment category }\end{array}$ \\
\hline & Image-guided biopsy \\
\hline $\begin{array}{l}\text { Locoregional staging for as- } \\
\text { sisting clinical TNM stage }\end{array}$ \\
\hline $\begin{array}{l}\text { For patients on active } \\
\text { surveillance }\end{array}$ & $\begin{array}{l}\text { Determine stability vs. } \\
\text { progression }\end{array}$ \\
\hline $\begin{array}{l}\text { For postoperative } \\
\text { patients }\end{array}$ & $\begin{array}{l}\text { Determine residual/recurrent } \\
\text { disease }\end{array}$ \\
\hline $\begin{array}{l}\text { For postradiotherapy } \\
\text { patients }\end{array}$ & $\begin{array}{l}\text { Determine postradiation } \\
\text { changes vs. recurrence }\end{array}$ \\
\hline
\end{tabular}

Abbreviations: CSC, clinically significant prostate cancer; mp-MRI, multiparametric magnetic resonance imaging; PI-RADS v2, prostate imaging reporting and data systems, version 2; TNM, tumor-lymph nodes-metastasis.

gland through which ejaculatory ducts traverse at the base of the prostate. It is relatively hypointense than the $\mathrm{PZ}$ and may occasionally show mild restricted diffusion. A vertical T2 dark strip in the anterior midline of the gland is called the anterior fibromuscular stromal zone (AFMSZ). A thin strip of compressed gland around the urethra, the periurethral zone is often indistinct on MRI. For standardized reporting as per PI-RADS v2, these zones are divided into sectors as per the sector map, adapted from the ESUR Prostate MRI guidelines 2012. ${ }^{17}$ It employs 39 sectors or regions: 36 for the prostate, 2 for the seminal vesicles, and 1 for the external urethral sphincter. On MRI, the prostate gland is divided superoinferiorly into three equal parts: base, mid-gland, and apex, each being divided into zones and sectors. For mapping, imaginary vertical and horizontal axes are drawn in the axial sections, dividing the zones into the left-right and anterior-posterior halves, respectively. The horizontal axes in the PZ continue obliquely. At the base, the posterocentral peripheral gland surrounding the ejaculatory ducts comprises the $\mathrm{CZ}$. This zone may be indistinct in the elderly. The bulk of the remaining gland comprises the PZ peripherally and the TZ centrally. At the mid-gland and apex, the posterior PZ is further divided into the posteromedial and the posterolateral sectors on each side. The AFMSZ is the anterior most sector in all three parts ( $\boldsymbol{- F i g .} \mathbf{1}$ ). There is no true prostatic capsule, but the fibromuscular tissue seen as a T2 dark rim posterior and posterolateral to the gland is considered the capsule and is a landmark for detecting extracapsular extension (ECE) in the form of a bulge or discontinuity. The neurovascular bundles are seen to course posterolateral to the prostate as linear thin T2 dark structures along the 5 and 7 o'clock axes. The seminal vesicles are seen as wing-like structures atop the base of the gland on either side; they are T2 hyperintense and have a multiloculated appearance. Medially, a T2 dark area is often seen, which is actually a part of the central zone with age-related changes and should not be confused for a tumor. 

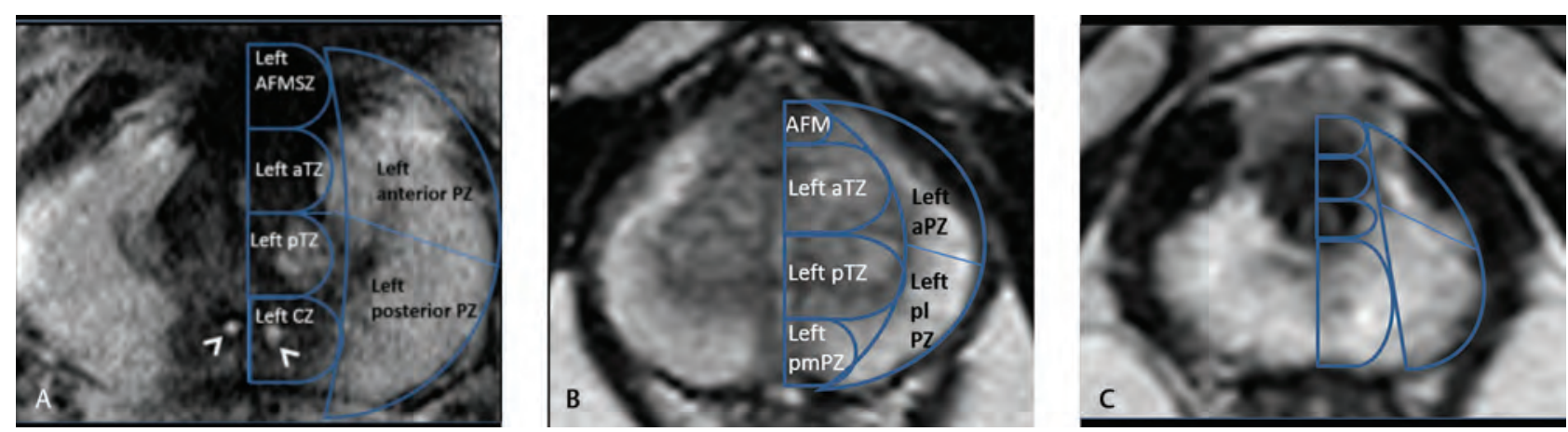

Fig. 1 Axial T2W images delineating sector map anatomy of prostate as per PI-RADS. (A) Base of prostate: The middle two blue semicircular areas represent TZ (transition zone) divided into anterior and posterior zones; Blue lenticular shape represents PZ (peripheral zone). These are divided horizontally into anterior and posterolateral zones/sectors. At the base, the posteromedial sectorial region is occupied by the CZ (central zone) outlined by the bottom blue semicircular area; the ejaculatory ducts coursing through the CZ are marked by arrowheads. The topmost sector marked in blue semicircular area in the anterior midline represents the AFMSZ (anterior fibromuscular stromal zone). (B) Mid-gland: TZ and PZ as described in $1 \mathrm{~A} ; \mathrm{CZ}$ is replaced by the posteromedial PZ. (C) Apex: TZ and PZ as described in $1 \mathrm{~A}$; CZ is replaced by the posteromedial PZ.

\section{Mp-MRI Protocol-PI-RADS v2 Centric Planning}

PI-RADS v2 is a clinically accepted, practical lexicon for categorizing prostate lesions from least to most suspicious. MRI protocols require to parallel PI-RADS categorization for optimal reporting and standardization.

- Timing: It was earlier suggested to wait for 6 weeks post biopsy for regression of hemorrhage before performing an mp-MRI. PI-RADS v2 states that MRI may be done anytime after biopsy if the aim is detection and characterization of cancer. This is because, while only the nonhemorrhagic areas can be well characterized on the MRI, the hemorrhagic areas are already sampled for histology. However, for staging, it recommends waiting for 6 weeks.

- $1.5 T$ versus 3T MRI: The minimal requirement for an mp-MRI is a 1.5T scanner. Both 1.5T and 3T field strengths provide diagnostic quality images when optimized scan protocols are used. ${ }^{18}$ 3T performs better with higher signal-to-noise ratio (SNR) (1.5-1.8 times that of 1.5T), providing higher resolution images. However, artifacts are more pronounced on 3T MRI, and hence $1.5 \mathrm{~T}$ may be preferred in presence of metallic implants/prosthesis, in an anatomical proximity to the prostate (e.g., hip prosthesis).

- Endorectal coil versus surface coil: Endorectal coils are not required when using a 3T scanner and are optional for a 1.5T scanner. Although a higher SNR is expected with an endorectal coil, the receiver profile is inhomogeneous and leads to greater signal loss with increasing distance. ${ }^{19}$ This is especially problematic in DWI, which is the cornerstone of a PI-RADS v2-centric protocol. Also, patient discomfort leads to motion artifacts, and cost escalation may further prohibit its routine use. Hence, surface array coils are preferred to endorectal coils in most cases.

- Prescan medication: Antispasmodic medications are optional. Rectal evacuation on the day of the procedure is helpful. Enema is avoided as it can cause increased peristalsis.

\section{Planning Sequences and Their Utility}

PI-RADS v2 pivots around three sequences: T2W, DWI, and DCE. Additional sequences, however, are required for a holistic assessment of the prostate and hence are included here, which are a large field of view (FOV), T1W, and T2W sequences for regional, nodal, and osseous assessment. Magnetic resonance spectroscopy (MRS) and perfusion kinetics are not supported by PI-RADS v2 and hence are not discussed ahead. The same localizer for small FOV T2W, DWI, and DCE should be used for better cross-referencing of findings.

- T2W: Slice thickness $3 \mathrm{~mm}$ without gap, same localizer as for DWI and DCE, FOV 12 to $20 \mathrm{~cm}$ to encompass the prostate and seminal vesicles, in-plane dimension $\leq 0.7 \mathrm{~mm}$ phase and $\leq 0.4 \mathrm{~mm}$ frequency. Small FOV T2W images are acquired in all three planes, sagittal, oblique axial and oblique coronal, along the axes of the prostate as seen on the localizer image ( $\mathbf{- F i g . ~ 2 ) . ~}$

The zonal differentiation of the prostate is best displayed on T2W sequence, and according to PI-RADS v2, transition zone cancers are best characterized on this sequence. The $\mathrm{PZ}$ appears hyperintense, $\mathrm{CZ}$ appears moderately hypointense, and $\mathrm{TZ}$ has variable signal intensity (SI) as described in anatomy. T2W also plays a critical role for detecting extraprostatic extension of disease.

- DWI: Free-breathing spin echo planar imaging (EPI) sequence with spectral fat saturation-TE $\leq 90$ milli-seconds, $\mathrm{TI}>3,000$ milliseconds, slice thickness $\leq 4 \mathrm{~mm}$ without gap, FOV 16 to $22 \mathrm{~cm}$, in-plane dimension $\leq 2.5 \mathrm{~mm}$ phase, and frequency. For the purpose of creating apparent diffusion coefficient (ADC) maps, if only two $\mathrm{b}$ values can be used, PI-RADS v2 recommends b values of 50 to $100 \mathrm{~s} / \mathrm{mm}^{2}$ and b value of 800 to $1,000 \mathrm{~s} / \mathrm{mm}^{2}$. PI-RADS v2 also recommends the inclusion of high $b$ value images $\left(b=1,400-2,000 \mathrm{~s} / \mathrm{mm}^{2}\right)$, computed from the low b value images or obtained directly as a separate acquisition (-Fig. $\mathbf{3}$ ). This is the most crucial sequence, 

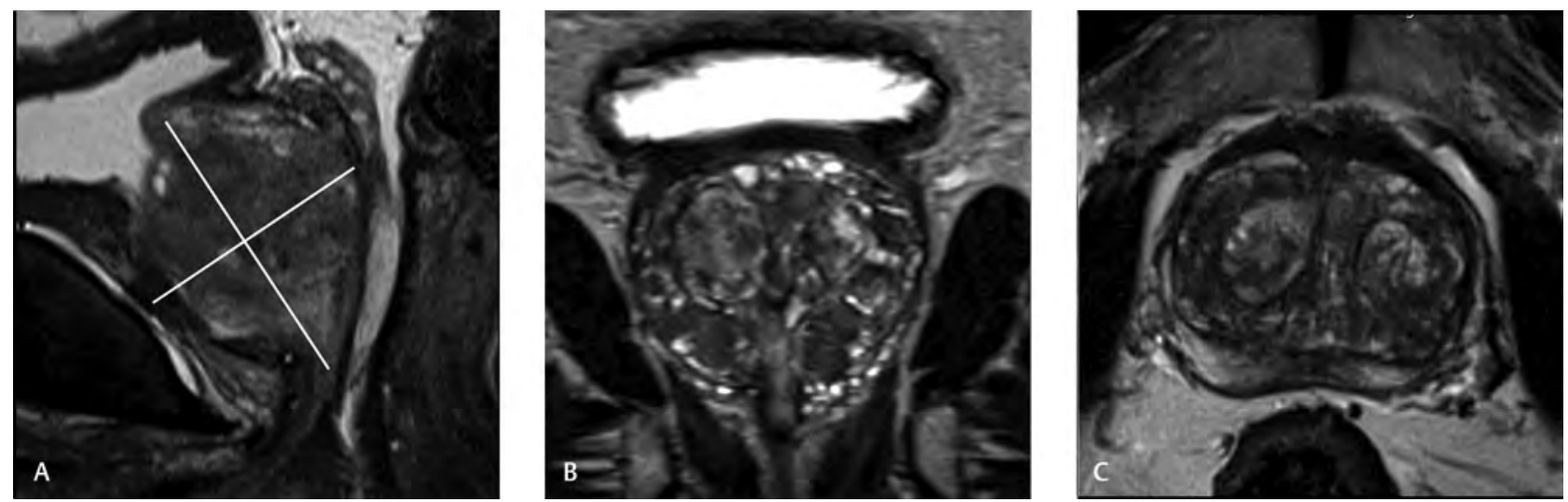

Fig. 2 Scanning protocol for T2 W images. (A): T2W sagittal image demonstrating planning of oblique coronal and oblique axial planes based on the orientation of gland. (B) Oblique coronal image. (C) Oblique axial image.
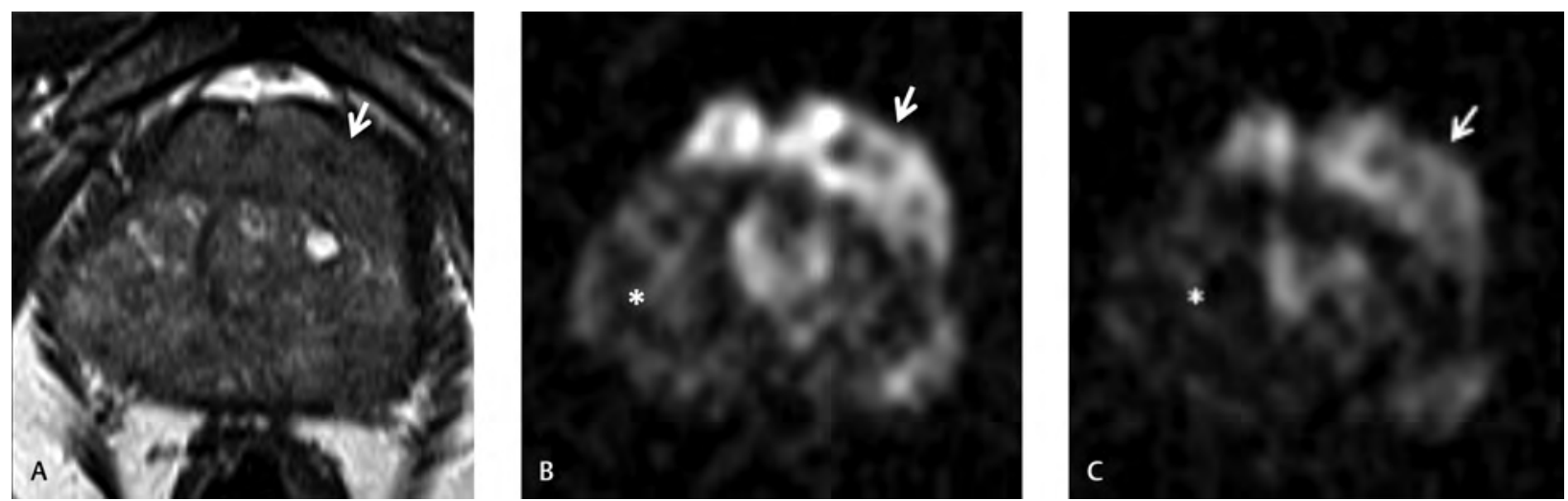

Fig. 3 Effect of high b value in diffusion-weighted imaging. (A) T2W axial demonstrates a moderately hypointense mass involving the anterior part of transitional zone and anterior fibromuscular stromal zone (white arrow). (B) Corresponding DWI axial $(b=1,400)$ demonstrates significant hyperintense signal (white arrow). White asterisk represents mild hyperintensity in right transition zone. (C) Corresponding DWI axial $(b=2,000)$ demonstrates the hyperintense signal (white arrow) in the mass is persistent, whereas the signal in the rest of the gland is suppressed.

especially for evaluation of the PZ where most cancers originate. ADC value of a tumor is inversely proportional to Gleason's grade. ADC below the range of $750-900 \mu \mathrm{m}^{2} / \mathrm{s}$ is considered an optimal cutoff.

- Dynamic contrast-enhanced scan (DCE): Temporal resolution $<10$ to $<15$ seconds, 2D or 3D T1 GRE (with 3D preferred over 2D), TR/TE: $<100$ milliseconds/ $<5$ milliseconds, for an observation rate of more than 2 minutes, IV gadolinium $0.1 \mathrm{mmol} / \mathrm{kg}$ at 2 to $3 \mathrm{~mL} / \mathrm{s}$ starting with continuous image data acquisition. DCE supports DWI in characterizing PI-RADS 3 lesions in the peripheral zone. PI-RADS v2 classifies DCE as positive when the lesion displays early and focal enhancement (the washout characteristics or graph are less important) ( - Fig. 4).

- T1W: T1W sequence is invaluable in diagnosing postbiopsy prostatic hemorrhage, which would otherwise falsely appear to enhance early and restrict diffusion, a combination that can mis-label hemorrhage as PI-RADS 5 lesion. Due to the anticoagulant property of citrate, noncancerous regions in the prostate are more likely to bleed compared to prostate cancer that has low citrate. This is known as the "hemorrhagic exclusion sign"20 (-Fig. 5). It is therefore useful to correctly diagnose hemorrhage with the help of a T1W sequence. A large FOV T1W sequence is also useful in diagnosing pelvic adenopathy and bony lesions.

\section{PI-RADS (Prostate Imaging Reporting and Data Systems)}

The concept of PI-RADS stems from the original concept of a standardized lexicon and reporting in breast imaging, the BIRADS system, introduced in the late 1980s..$^{21}$ PI-RADS is a homogenized reporting system in prostate imaging that serves as a guide for further clinical management. Before PI-RADS, the heterogeneity of MRI prostate acquisition and reporting did not favor clinical acceptance of MRI in the algorithm of prostate cancer diagnosis or staging. This led to the establishment of PI-RADS and its subsequent revision to v2, with credit to the combined efforts of ACR, ESUR, and the AdMe Tech Foundation. The purpose of PI-RADS and its assessment categories are listed in - Table 2 .

The normal appearance of the gland is described earlier in the article. To report CSC, it is pertinent to understand pathologic features of the gland, to differentiate benign morphologies from malignancy. - Table 3 summarizes commonly encountered benign prostatic pathologies and its morphologic description on MRI.

PI-RADS v2 assessment revolves around three sequences: DWI, T2W, and supplementary DCE sequence. A simplified mental algorithm for a radiologist is suggested for the PZ and TZ as charted in -Figs. 11 and 13, 

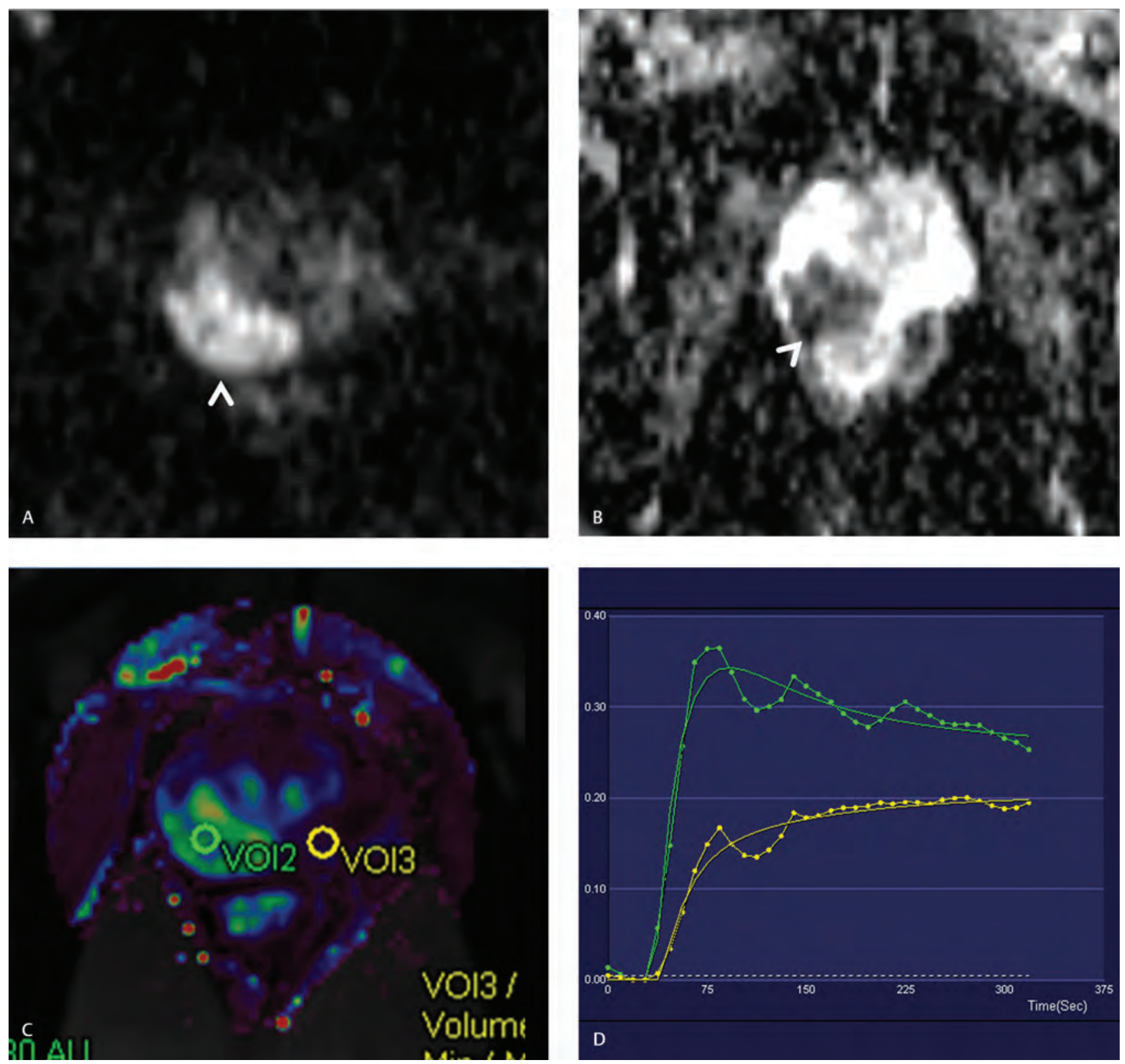

Fig. 4 Dynamic contrast imaging (DCE). (A) DWI axial image $(b=2,000)$ shows hyperintense lesion in the right peripheral zone at the base (white arrowhead). (B) Corresponding ADC image in axial plane shows restricted diffusion (white arrowhead). (C) Corresponding DCE color map in axial plane shows hypervascular enhancement, seen as a differential green hue, that is, positive DCE (VOI2) in right peripheral zone as compared with the left peripheral zone (VOI3). (D) Qualitative assessment of the contrast wash-in characteristics: Signal intensity curve from the lesion in right peripheral zone (green curve) is type $C$ with shorter time to peak and higher peak enhancement, consistent with a graphic representation of positive DCE as compared with left side (yellow curve) type A curve, which is a negative DCE. Washout analysis is not a feature of PI-RADS assessment.

respectively, to navigate through the mp-MRI sequences to reach an accurate PI-RADS assessment category. - Table 4 guides assessment score based on the findings seen on DWI and T2W sequences.

Peripheral zone: The PZ is assessed primarily by DWI and when indeterminate (PI-RADS3) is supported by DCE. A positive DCE will upscore the lesion to PI-RADS4, whereas a negative DCE will continue to let the score remain as PI-RADS3 (-Fig. 6). When DWI is suboptimal, the assessment of PZ may be based on the T2W imaging findings, if repeating DWI is not feasible ( - Fig. 7). - Fig. 8 cites an example of positive DCE upscoring a DWI-based indeterminate PI-RADS
3 assessment to PI-RADS 4 (-Fig. 8). - Figs. 9 and $\mathbf{1 0}$ cite an example of PI-RADS 4 and 5 lesions in the PZ.

Transitional zone: The TZ is assessed primarily by T2W (memory aid: "T for T") and, when indeterminate (PI-RADS 3), is supported by DWI. DCE does not help characterize lesions in the TZ, due to frequent heterogenous pattern of enhancement encountered in the TZ ( $\boldsymbol{- F i g . 1 2}$ ).

PI-RADS v2 is a guide for assessment of CSC. However, once the radiologist sees a suspicious focal lesion, locoregional staging of the tumor should also be provided. The American Joint Cancer Committee clinical TNM (tumorlymph node-metastasis) staging 8th edition relies on 

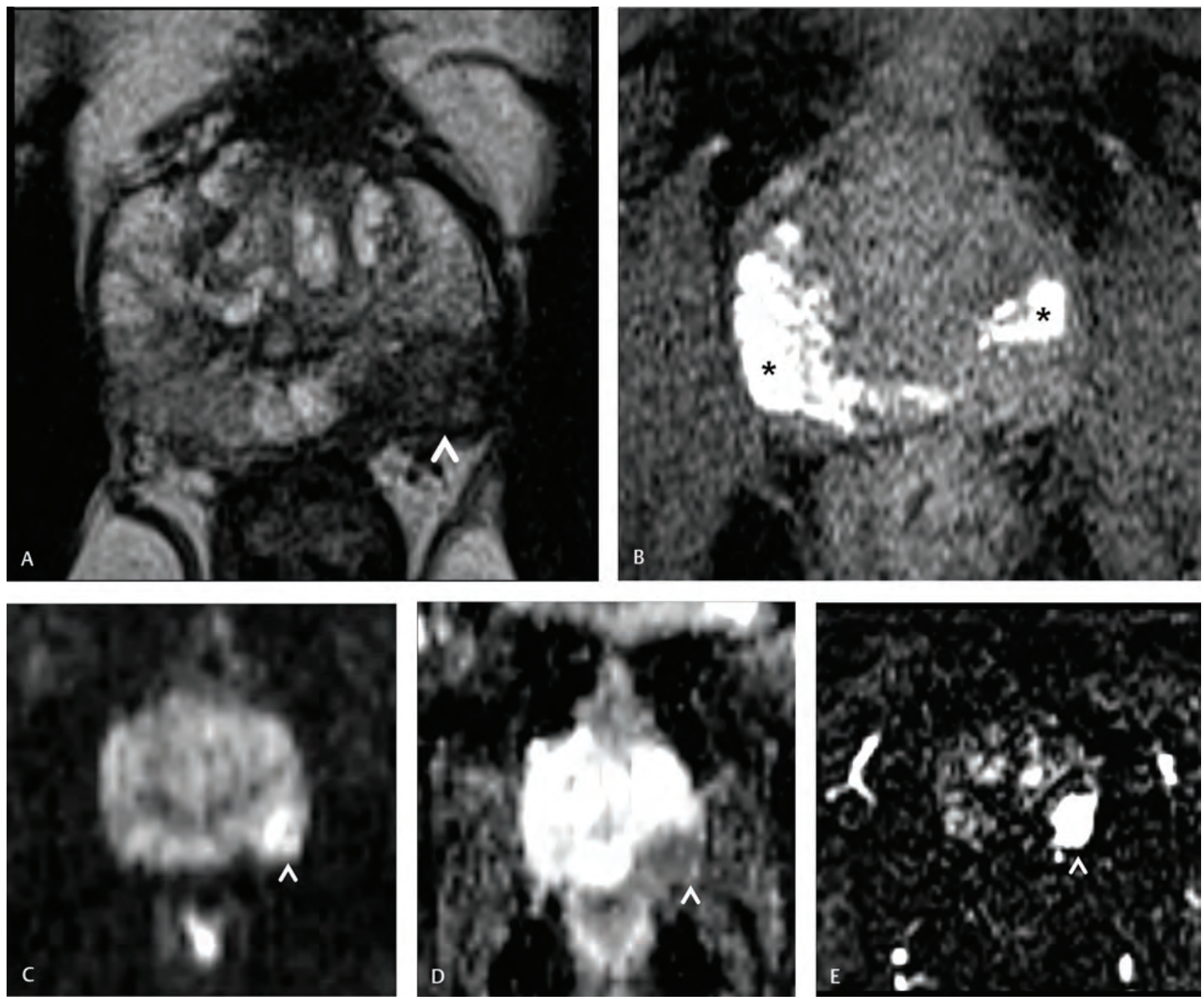

Fig. 5 Hemorrhagic exclusion sign-postbiopsy MRI of prostate cancer. (A) T2 axial demonstrates hypointense lesion in the left peripheral zone base (white arrowhead) with bulging capsular margin. (B) Corresponding T1W FS axial demonstrates hyperintensity representing early subacute hemorrhage due to previous biopsy (black asterisk) with sparing of left base peripheral lesion. (C) Corresponding DWI axial image $(b=1,000)$ shows hyperintense lesion in the left base (white arrowhead). (D) Corresponding ADC image shows significant restricted diffusion. (E) Corresponding subtracted postcontrast dynamic image in axial plane shows hypervascular enhancement of the lesion (white arrowhead).

findings of digital rectal examination, for organ confined nodules and those with extraprostatic extension because mp-MRI is not yet standardized globally.22 However, when MRI is performed, an organ-confined disease is marked as T2 ( $a, b$, and $c$ for $<1 / 2$ lobe, $>1 / 2$ lobe and both lobes, respectively) (-Fig. 14); EPE as T3a and seminal vesicle invasion as T3b ( - Fig. 15). Regional adenopathy comprises pelvic nodes below the bifurcation of the common iliac artery and excludes inguinal nodes. M1a, M1b, and M1c represent local aggressive extent, bony metastases, and distant metastases, respectively.

- Table 5 provides a suggested reporting checklist for comprehensive prostate magnetic resonance reporting incorporating both PI-RADS and staging.

\section{Pitfalls of PI-RADS}

There are certain pitfalls of PI-RADS v2 that must be avoided. A careful evaluation of the T2W sequence is helpful in analyzing a PZ finding of restricted diffusion before scoring as P5. A mental checklist for this will include to rule out tiny hyperintense areas within or around a well circumscribed such nodule, in which case it would favor an extruded BPH nodule from the TZ into the PZ. Another is to look for symmetry of the findings and a correlation with the coronal plane in differentiating a compressed $\mathrm{CZ}$ (which has a lower SI than the $\mathrm{PZ}$ and may show mild restriction in diffusion), from a true lesion, particularly with coexisting changes of BPH. Bilaterally in the posterolateral PZ, the neurovascular bundle may be close to the capsule of the gland or project into the $\mathrm{PZ}$ of the gland, with false restricted diffusion. A correlate with T2W finding of higher SI with differential variation in enhancement on DCE can help ruling out a P5 lesion. The AFMSZ may occasionally have a false low ADC due to dark SI on T2W sequence. Lack of an intermediate SI lesion at this site on T2W sequence, along with a negative DCE, can downscore the finding to P2. Prostatitis may mimic CSC with ECE and usually requires careful history correlation. Granulomatous prostatitis, 
Table 2 Indication for PI-RADS and assessment categories

\begin{tabular}{|c|c|}
\hline \multicolumn{2}{|c|}{ Need for a PI-RADS system } \\
\hline \multicolumn{2}{|c|}{$\begin{array}{l}\text { Improve detection, localization, characterization, and risk } \\
\text { stratification in patients with suspected cancer in treatment- } \\
\text { naïve prostate gland, and improve patient outcome }\end{array}$} \\
\hline \multicolumn{2}{|c|}{$\begin{array}{l}\text { Establish technical parameters; standardize terminology } \\
\text { and reports }\end{array}$} \\
\hline \multicolumn{2}{|c|}{$\begin{array}{l}\text { Develop assessment categories to aid in management } \\
\text { to triage as normal, active surveillance or immediate } \\
\text { intervention and facilitate-targeted biopsy }\end{array}$} \\
\hline \multicolumn{2}{|c|}{$\begin{array}{l}\text { Enable data collection and outcome monitoring; educate } \\
\text { radiologists to reduce variability and enhance multidiscipli- } \\
\text { nary communications }\end{array}$} \\
\hline \multicolumn{2}{|c|}{ PI-RADS assessment categories } \\
\hline PI-RADS 1 & Very low (CSC is highly unlikely to be present) \\
\hline PI-RADS 2 & Low (CSC is unlikely to be present) \\
\hline PI-RADS 3 & Intermediate (presence of CSC is equivocal) \\
\hline PI-RADS 4 & High (CSC is likely to be present) \\
\hline PI-RADS 5 & Very high (CSC is highly likely to be present) \\
\hline
\end{tabular}

Abbreviations: CSC, clinically significant prostate cancer; PI-RADS, prostate imaging reporting and data systems.

\section{Role of MRI in Personalized Management of Prostate Cancers}

Mp-MRI with PI-RADS 3 assessment category, in combination with clinical parameters, plays a pivotal role in active surveillance. MRI is useful for the detection of clinically significant disease at the initial assessment of men considering active surveillance. To use MRI as a monitoring tool in surveillance, it will be necessary to define both radiologic significance and radiologic progression. ${ }^{24}$ Radical prostatectomy data show that positive MRI is more likely than a negative MRI to be associated with upgrading (Gleason's score > $3+3$ ) ( $43 \%$ vs. $27 \%$ ) of disease rather than increasing the T-stage of disease. Hence it is a valuable adjunct to PSA in patients on active surveillance. ${ }^{8}$ Fairly accurate locoregional extent on an MRI helps in therapy planning. An organ-confined disease or one with minimal ECE can be operated by robotic surgeries, whereas more extensive disease may require open surgery. It also assists in planning nerve-sparing surgeries. ${ }^{25,26}$ MRI plays a role in radiation planning for contouring and boost delivery to the target lesions. ${ }^{27}$ Restricted diffusion and intermediate T2 SI, when present, are reliable surrogate

Table 3 Morphologic assessment of benign prostatic pathologies

\begin{tabular}{|c|c|c|c|}
\hline $\begin{array}{l}\text { Benign } \\
\text { pathologies }\end{array}$ & $\begin{array}{l}\text { Choice of } \\
\text { sequence }\end{array}$ & Morphology & Remarks \\
\hline $\mathrm{BPH}$ & $\begin{array}{l}\text { T2W } \\
\text { If doubtful, then } \\
\text { DWI and ADC }\end{array}$ & $\begin{array}{l}\text { Well-circumscribed, capsulat- } \\
\text { ed nodules with heterogenous } \\
\text { signal intensity and inter- } \\
\text { spersed cystic changes } \\
\text { Unlikely to show significant } \\
\text { restricted diffusion. }\end{array}$ & $\begin{array}{l}\text { A soap bubble-like appearance. } \\
\text { Occasionally, an exophytic nodular component of BPH } \\
\text { nodule may extrude into PZ. } \\
\text { If mild restricted diffusion is seen, repeating with higher } \\
\text { b value at } 1,500 \text { or } 2,000 \text {, may eliminate the spurious } \\
\text { finding. }\end{array}$ \\
\hline Prostatitis & $\begin{array}{l}\text { T2W } \\
\text { If doubtful, then } \\
\text { DWI/ADC in TZ } \\
\text { and DCE in PZ }\end{array}$ & $\begin{array}{l}\text { Ill-defined low signal intensity } \\
\text { on T2 without corresponding } \\
\text { significantly low ADC values }\end{array}$ & $\begin{array}{l}\text { Usually seen as streaks and bands. } \\
\text { Often seen in diabetics; may present clinically with high } \\
\text { PSA levels. }\end{array}$ \\
\hline Hemorrhage & T1W and T2W & $\begin{array}{l}\text { Often bright on both sequences. } \\
\text { But may vary depending on the } \\
\text { time of hemorrhage. }\end{array}$ & $\begin{array}{l}\text { Most common finding in postbiopsy setting. May persist } \\
\text { for several months. Gives a pseudo early enhancement. } \\
\text { Prudent to look at T1W in every case of prostate MR. } \\
\text { To carefully look for hemorrhage exclusion sign, sug- } \\
\text { gesting underlying cancer. }\end{array}$ \\
\hline Cysts & $\mathrm{T} 1 \mathrm{~W}$ and $\mathrm{T} 2 \mathrm{~W}$ & T1 dark and T2 bright. & Occasionally seen. \\
\hline Calcifications & T1W and T2W & $\begin{array}{l}\text { Usually dark but may be vari- } \\
\text { ably intermediate. }\end{array}$ & Occasionally seen. \\
\hline $\begin{array}{l}\text { Atrophy and } \\
\text { fibrosis }\end{array}$ & T1W and T2W & Usually hypointense on both. & A common finding seen in postradiotherapy cases. \\
\hline
\end{tabular}

Abbreviations: ADC, apparent diffusion coefficient; BPH, benign prostatic hyperplasia; CSC, clinically significant prostate cancer; DCE, dynamic contrast-enhanced; DWI, diffusion-weighted imaging; PSA, prostate-specific antigen; PZ, peripheral zone; PI-RADS, prostate imaging reporting and data systems; T1W, T1-weighted; T2W, T2-weighted; TZ, transition zone.

if idiopathic with no supportive clinical history such as Bacillus Calmette-Guerin (BCG) instillation for bladder cancer, tuberculosis, etc., may mimic a P4/5 lesion. An abscess, however, may be ruled out by its typical peripheral enhancement. A glimpse at the T1W sequence is necessary to exclude hemorrhage in all cases. Atrophy, calcifications, and necrosis are usually not confused with suspicious lesions when PI-RADS v2 is systematically followed ${ }^{20,23}$ ( - Fig. 16). markers of recurrence in a posttherapy patient, particularly post-radiotherapy.

\section{Scope for Further Research and Standardization in Magnetic Resonance Prostate Imaging}

PI-RADS v2 is aimed at global homogenization. Further inputs from trials of recent techniques will serve as a basis for development of future versions, aimed at overcoming 
Table 4 Assessment score based on DWI and T2W sequences

\begin{tabular}{|c|c|c|c|c|c|}
\hline $\begin{array}{l}\text { DWI-ADC } \\
\text { score }\end{array}$ & $\mathrm{PZ}$ and $\mathrm{TZ}$ & $\begin{array}{l}\text { T2W } \\
\text { score }\end{array}$ & TZ & $\begin{array}{l}\text { T2W } \\
\text { score }\end{array}$ & $\begin{array}{l}\text { PZ; as a rescue in absence of } \\
\text { good DWI }\end{array}$ \\
\hline 1 & Normal & 1 & $\begin{array}{l}\text { Homogeneous intermediate SI } \\
\text { (normal) }\end{array}$ & 1 & Uniform high SI (normal) \\
\hline 2 & $\begin{array}{l}\text { Indistinctly } \\
\text { hypointense }\end{array}$ & 2 & $\begin{array}{l}\text { Circumscribed low SI or heteroge- } \\
\text { neous encapsulated nodule (BPH) }\end{array}$ & 2 & $\begin{array}{l}\text { Linear/wedge-shaped low SI/indis- } \\
\text { tinct margin/diffuse mild low SI }\end{array}$ \\
\hline 3 & $\begin{array}{l}\text { Mild to moder- } \\
\text { ately hypointense }\end{array}$ & 3 & $\begin{array}{l}\text { Heterogeneous SI with obscured } \\
\text { margins (other than } 2,4 \text {, or } 5 \text { ) }\end{array}$ & 3 & $\begin{array}{l}\text { Heterogeneous SI or noncircum- } \\
\text { scribed, rounded, moderate low SI } \\
\text { (other than } 2,4 \text {, or } 5 \text { ) }\end{array}$ \\
\hline 4 & $\begin{array}{l}\text { Markedly } \\
\text { hypointense }< \\
1.5 \mathrm{~cm}\end{array}$ & 4 & $\begin{array}{l}\text { Lenticular or noncircumscribed, } \\
\text { homogeneous, moderately low SI, } \\
\text { and }<1.5 \mathrm{~cm}\end{array}$ & 4 & $\begin{array}{l}\text { Circumscribed, homogenous } \\
\text { moderate low SI focus/mass con- } \\
\text { fined to prostate and }<1.5 \mathrm{~cm}\end{array}$ \\
\hline 5 & $\begin{array}{l}\text { Markedly } \\
\text { hypointense > } \\
1.5 \mathrm{~cm} \text {, or with ECE }\end{array}$ & 5 & $\begin{array}{l}\text { Same as } 4 \text {, but } \geq 1.5 \mathrm{~cm} \text { or definite } \\
\text { ECE }\end{array}$ & 5 & $\begin{array}{l}\text { Same as } 4 \text { but } \geq 1.5 \mathrm{~cm} \text { or definite } \\
\text { ECE }\end{array}$ \\
\hline
\end{tabular}

Abbreviations: ADC, apparent diffusion coefficient; $\mathrm{BPH}$, benign prostatic hyperplasia; DWI, diffusion-weighted imaging; ECE, extracapsular extension; PZ, peripheral zone; SI, signal intensity; T2W, T2-weighted; TZ, transition zone.
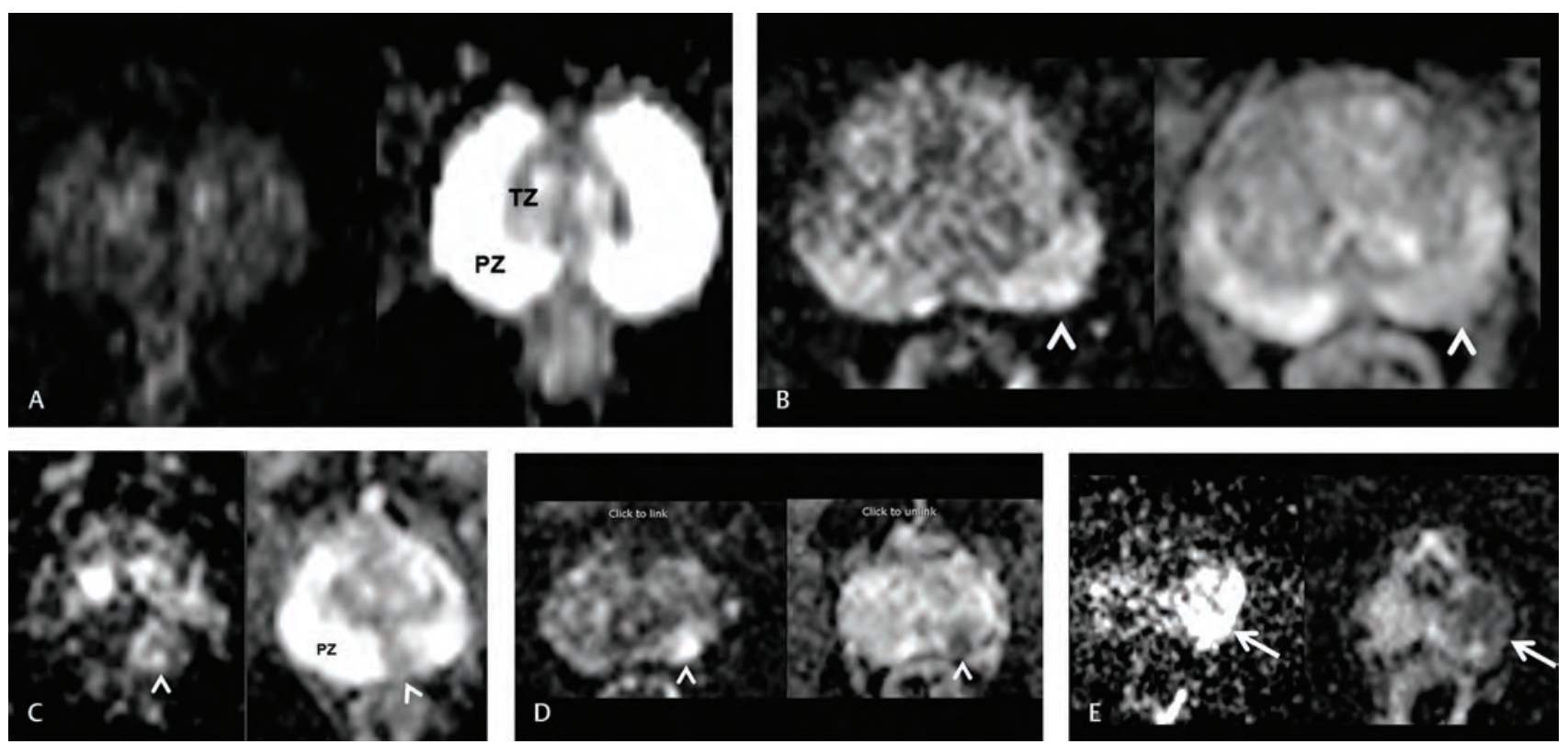

Fig. 6 PI-RADS scoring in peripheral zone (PZ) on DWI (left) and ADC (right) axial images (set of two images). (A) PI-RADS 1-Mild hyperintense signal of the gland on b-2000 DWI image; corresponding ADC image shows hyperintense signal in the PZ (TZ is transition zone). (B) PI-RADS 2-Subtle hyperintense signal on b-2000 DWI image in the left PZ (white arrowhead) without significant restrict diffusion on ADC (white arrowhead). (C) PI-RADS 3-Focal hyperintense signal on b-2000 DWI image in left PZ (white arrowhead) with mild restricted diffusion on ADC (white arrowhead). (D) P-IRADS 4-Focal hyperintense signal lesion on b-2000 DWI image in left PZ (white arrowhead) with significant restricted diffusion on ADC measuring $<1.5 \mathrm{~cm}$ (white arrowhead). (E) PI-RADS 5-Focal hyperintense signal lesion on b-2000 DWI image in left PZ at the apex measuring $>1.5 \mathrm{~cm}$ (white arrow) with significant restricted diffusion on ADC (white arrow).

the limitations of v2 and lowering false negativity. For this reason, advanced research tools beyond the scope of PI-RADS, such as MRS, diffusion tensor imaging (DTI), diffusional kurtosis imaging (DKI), multiple b-value assessment of fractional ADC, intravoxel incoherent motion (IVIM), blood oxygenation level-dependent (BOLD) imaging, and intravenous ultra-small super paramagnetic iron oxide (USPIO) agents, may be used based on institutional infrastructure and assist in future validation. $^{28,29}$ PSMA-PET-MRI can add molecular information to an MRI with fusion technology, delineating suspicious lesions for targeted biopsies following a negative biopsy in persistently elevated serum PSA and detecting local or metastatic lesions in biochemically recurrent prostate cancer and in personalized management for radiotherapy planning. ${ }^{29}$

\section{Conclusion}

The cornerstone of MRI prostate is the standardized and reproducible detection of clinically significant cancer. There is tremendous scope for novel applications such as computer-aided technology, pharmacodynamic sequences, and artificial intelligence that need robust development. 

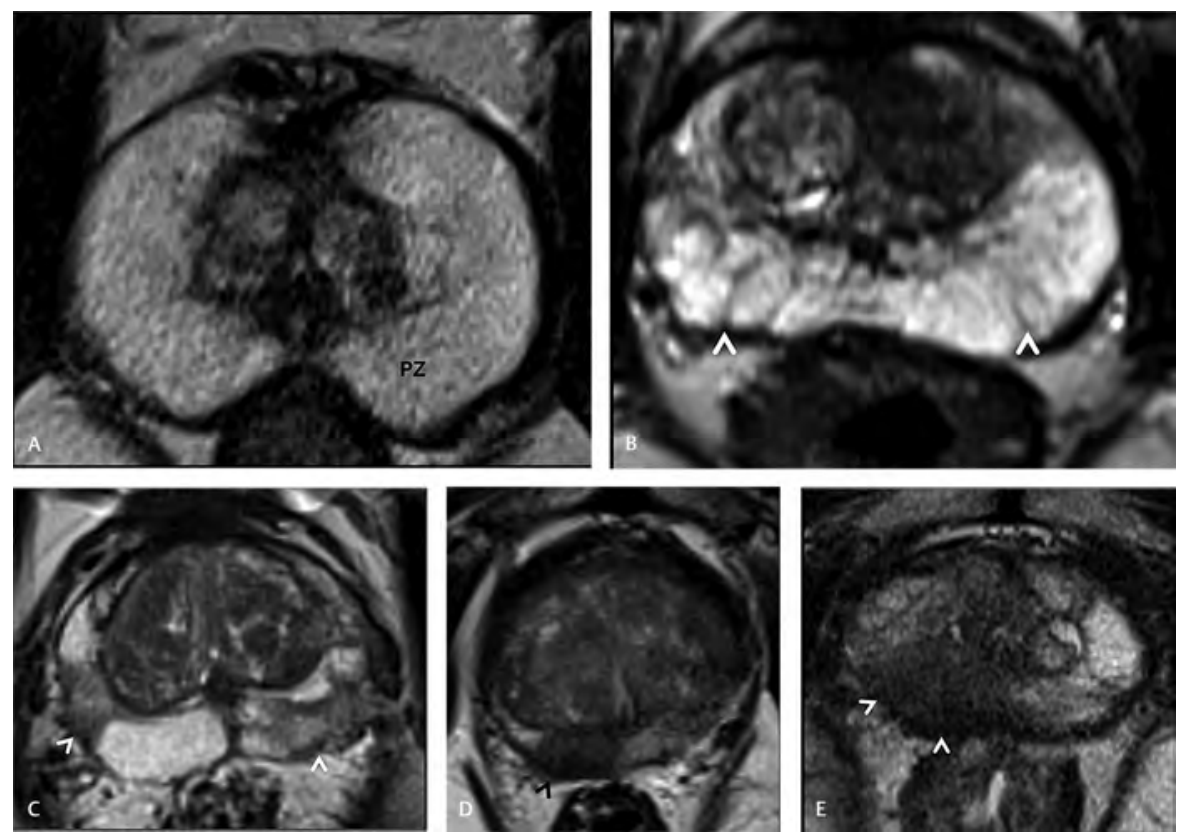

Fig. 7 PIRADS scoring in peripheral zone (PZ) on T2W axial images. (A) PIRADS 1-Homogeneous hyperintensity of the PZ. (B) PIRADS 2-PZ on both sides show linear hypointense strands (white arrowheads). (C) PIRADS 3-Noncircumscribed patchy mildly hypointense foci on both sides (white arrowheads). Some degree of volume loss is seen, probably a sequel to previous prostatitis. (D) PIRADS 4-Right PZ demonstrates circumscribed moderately hypointense nodule (black arrowhead) and is $<1.5 \mathrm{~cm}$. (E) PIRADS 5-Right PZ demonstrates moderately hypointense $>1.5-\mathrm{cm}$ mass (white arrowheads) with capsular bulge.
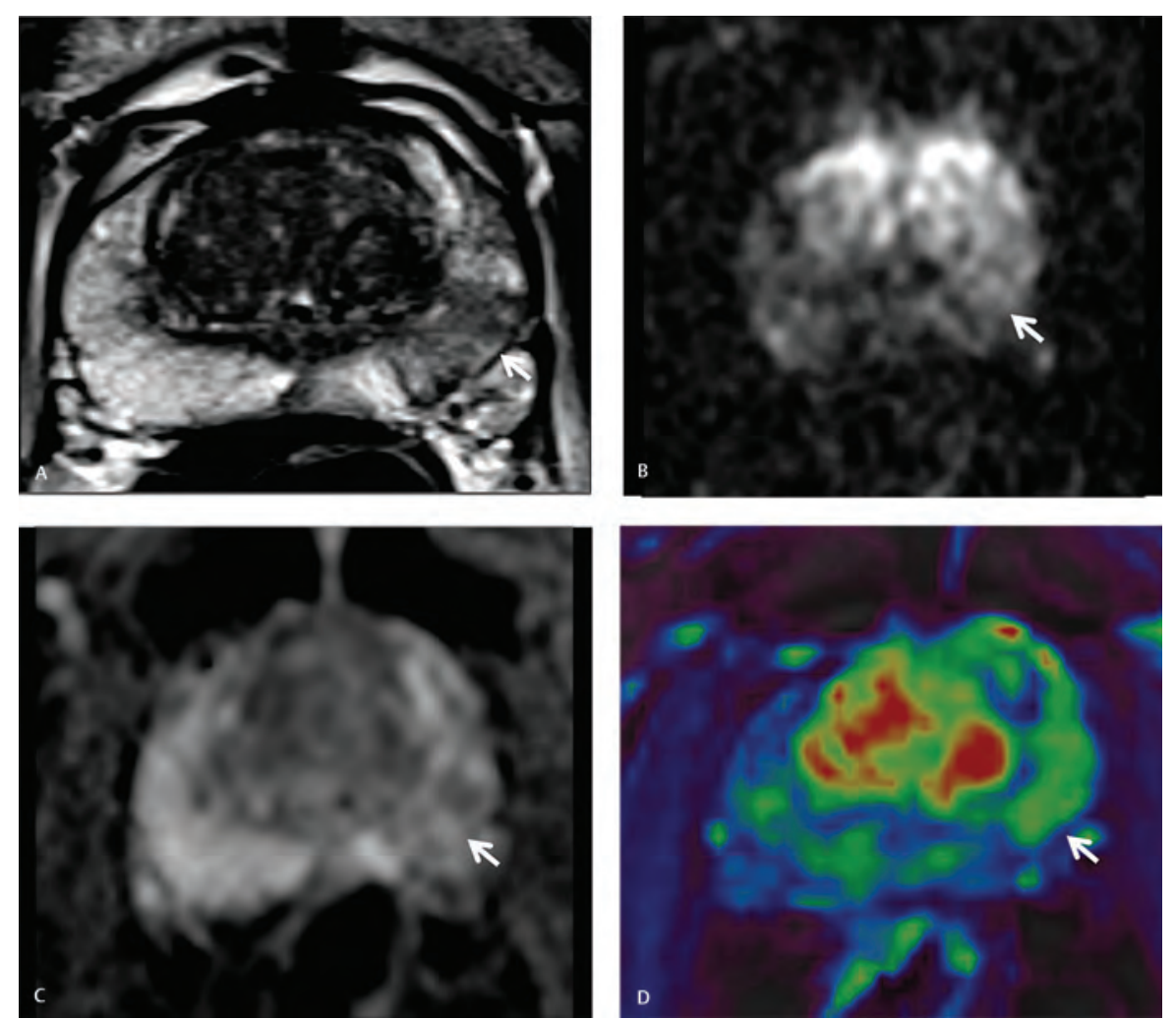

Fig. 8 PIRADS 3 lesion in peripheral zone (PZ) upgraded to PI-RADS 4. (A) T2W axial image demonstrates non circumscribed hypointense lesion in the left peripheral zone at the base (white arrow). (B) Corresponding DWI axial image $(b=1,000)$ demonstrates subtle hyperintensity (white arrow). (C) Corresponding ADC image demonstrates mild restricted diffusion (white arrow). (D) Corresponding dynamic contrast image demonstrates positive DCE (white arrow), shown as green color. Biopsy Histology-prostate cancer with Gleason's score of $3+4=7$.

Meanwhile improving our current standards, using a mental algorithm to navigate through mp-MRI sequences to achieve an accurate PI-RADS assessment, and keeping the pitfalls in mind will serve as a good database for future version upgrades, for better diagnostic outcomes and further improvement in clinical acceptance. 

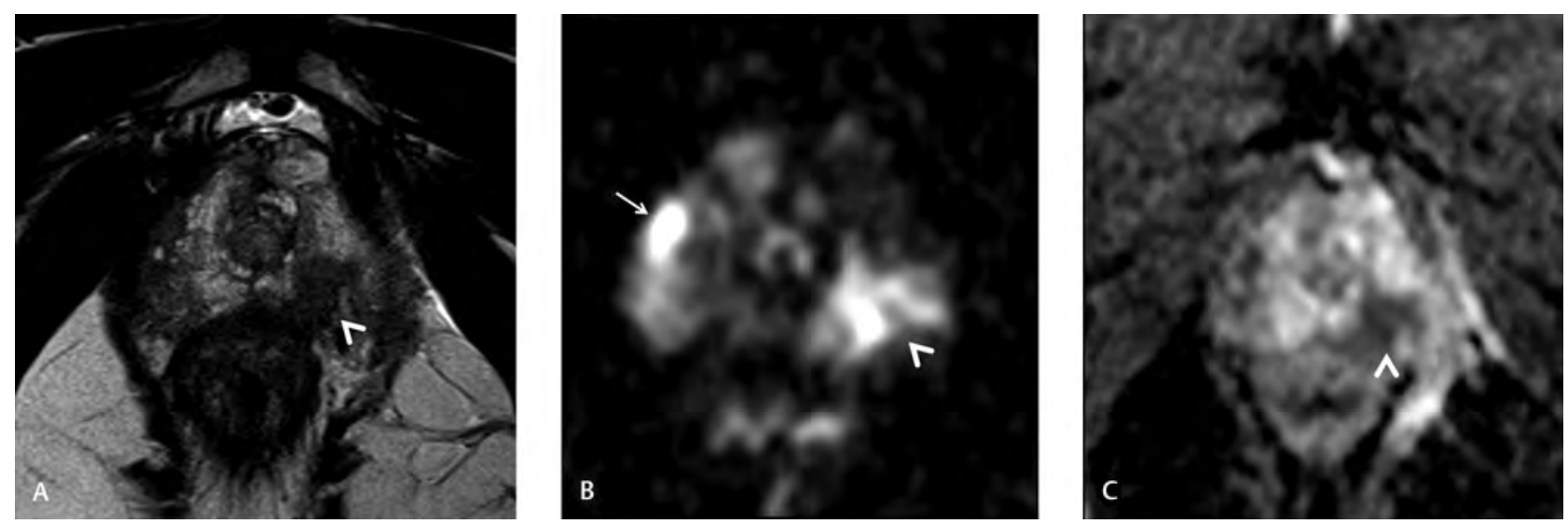

Fig. 9 PIRADS 4 lesion in peripheral zone (PZ). (A) T2W axial image demonstrates a moderate hypointense lesion in the left peripheral zone at the apex (white arrowhead). (B) Corresponding DWI image $(b=2,000)$ shows moderate hyperintensity (white arrowhead). A small focus of hyperintensity is also seen in the right peripheral zone. (C) Corresponding ADC image shows significant restricted diffusion (white arrowhead) and measures $<1.5 \mathrm{~cm}$. Another tiny right PZ lesion also shows restricted diffusion. Biopsy histology-prostate cancer with Gleason's score of $4+4=8$.
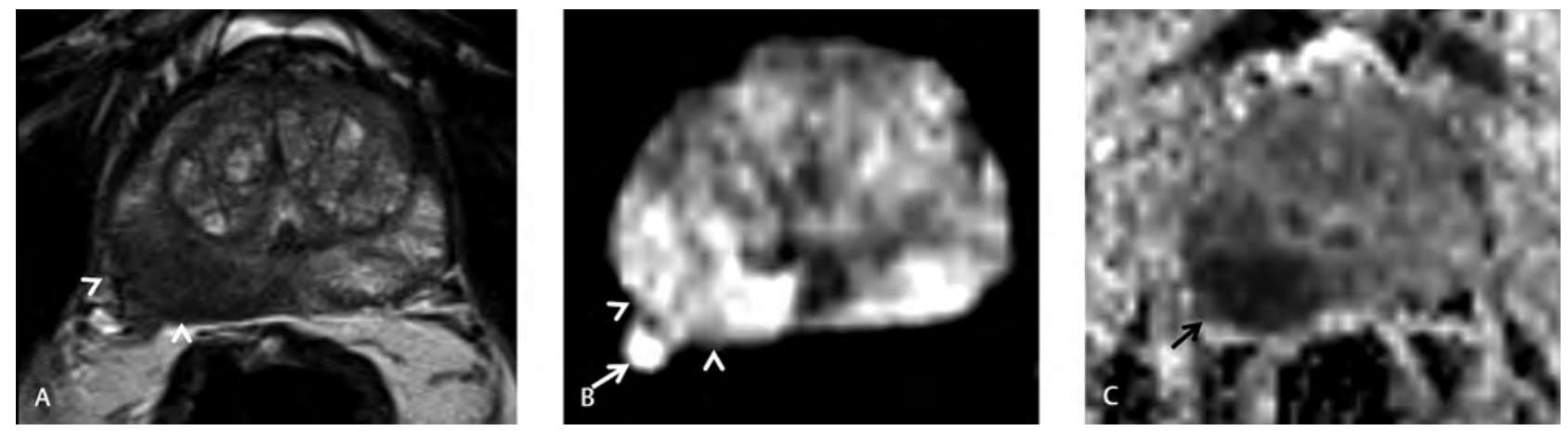

Fig.10 PIRADS 5 lesion in peripheral zone (PZ). (A) T2W axial image demonstrates a $>1.5 \mathrm{~cm}$ moderate hypointense lesion in the right peripheral zone at the base with irregular and bulging margins (white arrowheads) with loss of adjacent plane. (B) Corresponding DWI image $(b=2000)$ shows significant hyperintensity (white arrowheads). White arrow represents focal extracapsular extension. (C) Corresponding ADC image shows significant restricted diffusion (black arrow). Biopsy histology-high grade prostate cancer with Gleason's score of $4+4=8$.

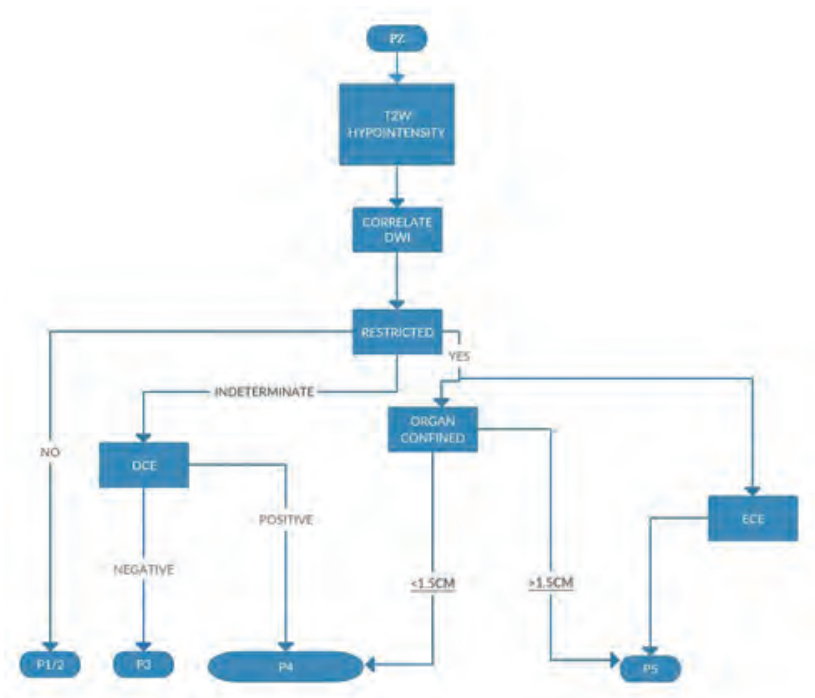

Fig. 11 Mental algorithm for evaluation of lesions in the peripheral zone. 

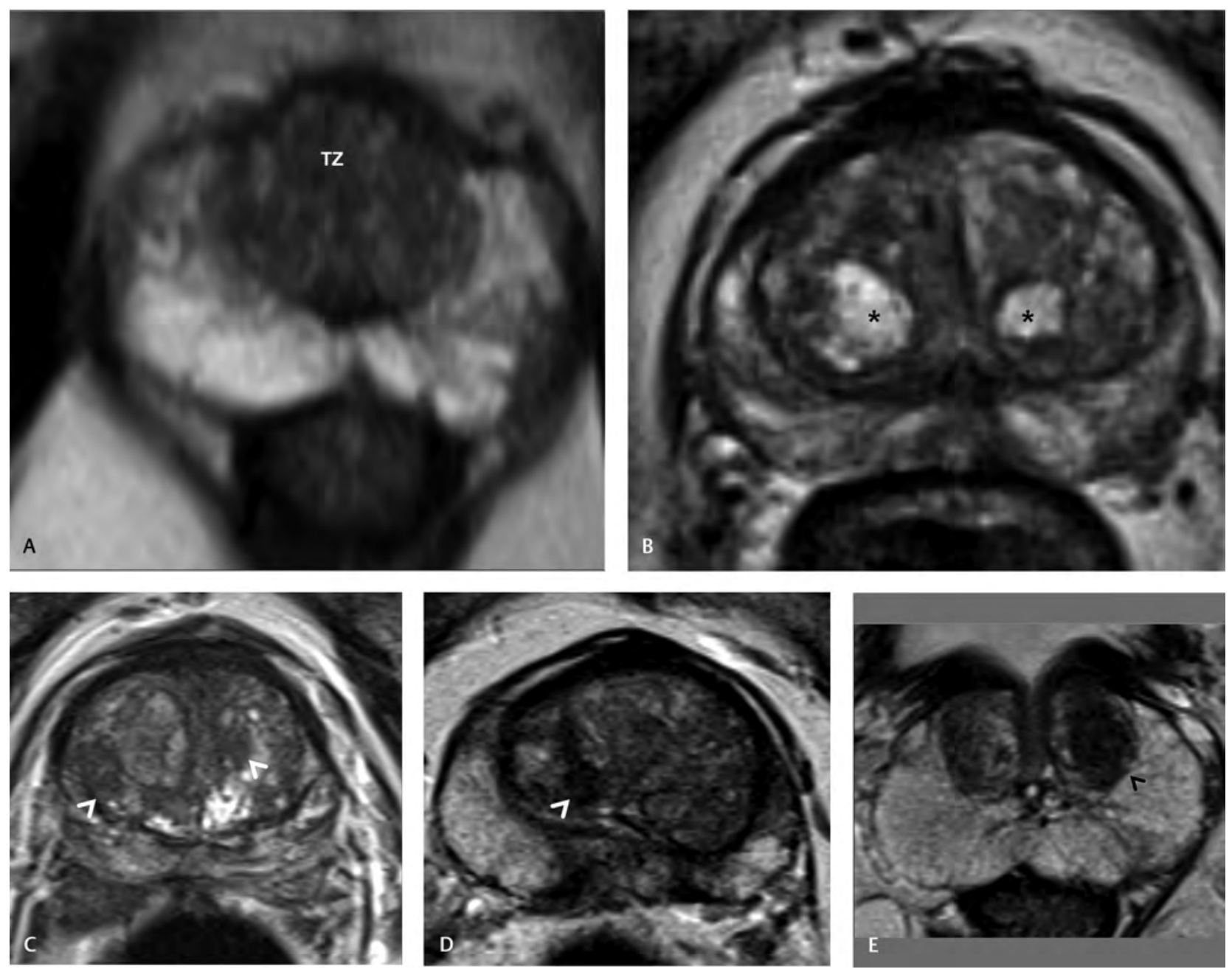

Fig. 12 PI-RADS scoring in transition zone (TZ) on T2W axial images. (A) PI-RADS 1-Heterogeneous appearance of normal size TZ. (B) PI-RADS 2-TZ is hypertrophied and shows well-capsulated T2 hyperintense nodules (black asterisk) giving a soap bubble like appearance. (C) PI-RADS 3-TZ is hypertrophied and shows non-circumscribed mildly hypointense foci on both sides (white arrowheads). (D) PI-RADS 4-TZ is hypertrophied with heterogeneous signal intensity. Right side shows moderate to marked hypointense nodule with noncircumscribed margins measuring $<1.5 \mathrm{~cm}$ (white arrowhead). (E) PI-RADS 5-Moderately hypointense noncapsulated nodule on left side measuring $>1.5 \mathrm{~cm}$ (black arrowhead).

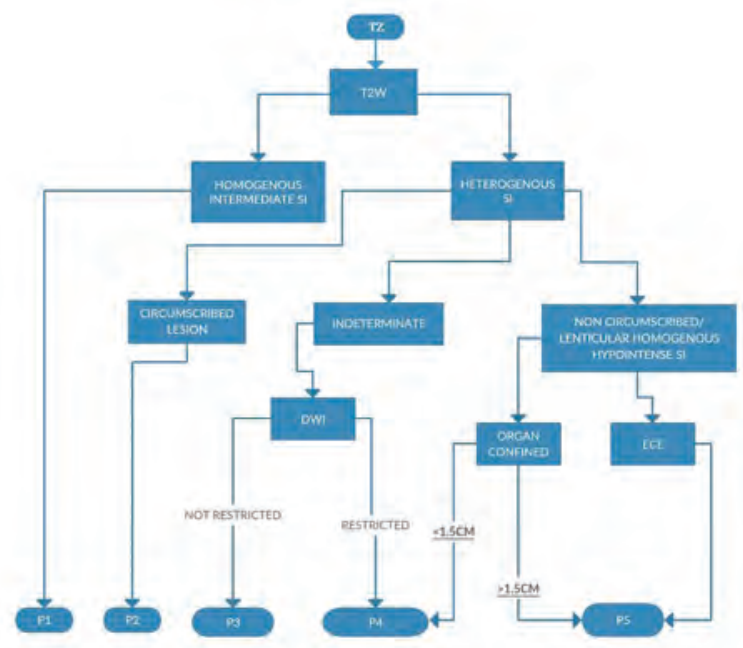

Fig. 13 Mental algorithm for evaluation of lesions in the transitional zone. 

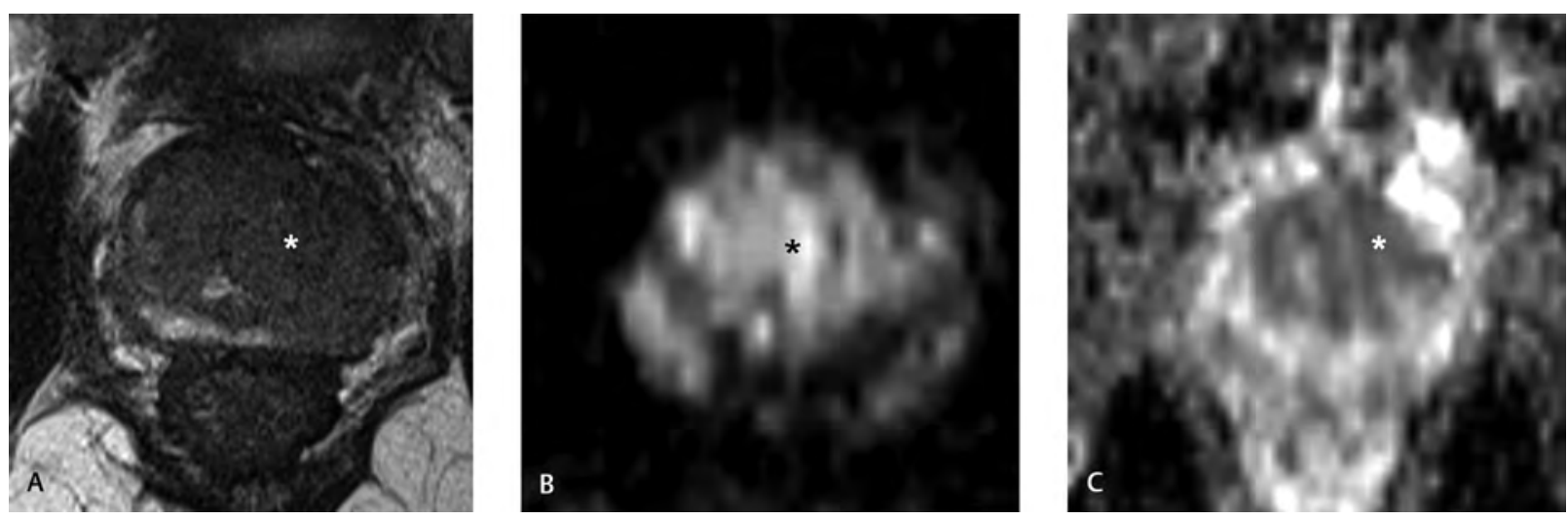

Fig. 14 Organ-confined PI-RADS 5 lesion involving bilateral transition zone (TZ). TNM 8th edition T-stage: T2c. (A) T2 axial image shows a moderate hypointense mass lesion in the left TZ (white asterisk) extending beyond midline. (B) Corresponding DWI axial image $(b=2,000)$ shows significant hyperintense signal (black asterisk). (C) Corresponding ADC image shows significant restricted diffusion (white asterisk). Biopsy histology-high-grade prostate cancer with Gleason's score of $4+5=9$.
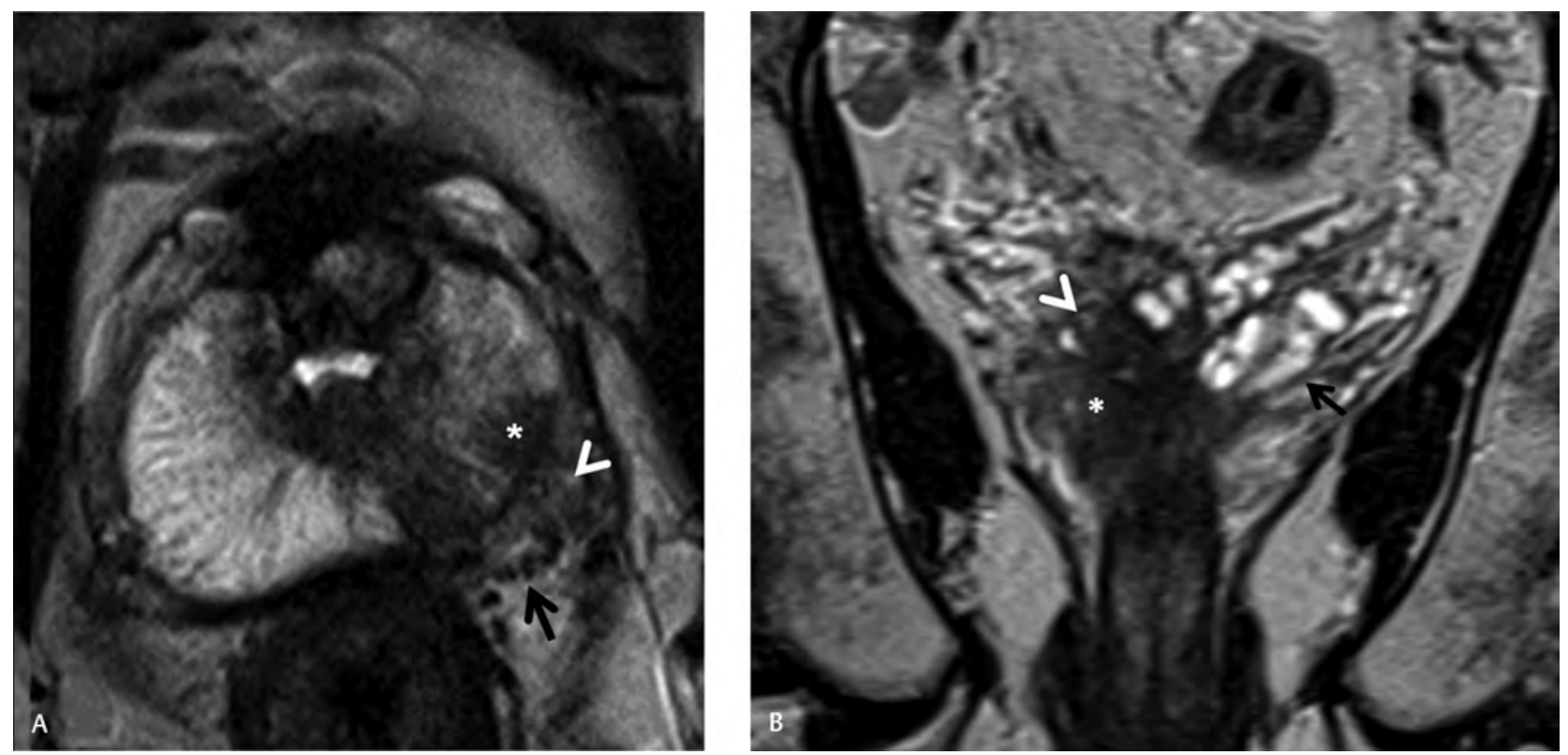

Fig. 15 Prostate cancer demonstrating extra-prostatic disease. TNM 8th edition T-stage: T3a. (A) T2 axial image demonstrates a hypointense lesion in the left peripheral zone at the base (white asterisk) with an irregular capsular margin and extension into the periprostatic fat (white arrow head). The fat plane with the neurovascular bundle is obliterated. Vessels are seen as round flow voids (black arrow). (B) T2 coronal image shows hypointense lesion in the right peripheral zone at the base (white asterisk) with encasement of the right seminal vesicle (white arrowhead). Black arrow points to the left seminal vesicle, which shows early involvement. 
Table 5 Reporting checklist

\begin{tabular}{|l|l|}
\hline Clinical profile & $\begin{array}{l}\text { DRE findings, PSA and PSA trend, biopsy findings if done (number of cores } \\
\text { positive, location, and Gleason's score) }\end{array}$ \\
\hline Prostate volume & $\begin{array}{l}\text { Measured by the ellipse formula: Max (AP diameter } \times \text { transverse diameter } \\
\times \text { longitudinal diameter) } \times 0.52\end{array}$ \\
\hline PSA density & \begin{tabular}{l} 
PSA/prostate volume \\
\hline Mapping lesion
\end{tabular} \\
\hline $\begin{array}{l}\text { Dimension (on T2W image for TZ and on DWI } \\
\text { for PZ) }\end{array}$ & $\begin{array}{l}\text { Largest axial dimension, if CC dimension is larger, to measure and report } \\
\text { the imaging plane }\end{array}$ \\
\hline Organ confined or ECE & \\
\hline Locoregional staging & \\
\hline Neurovascular bundle & Regional: Pelvic nodes \\
\hline Seminal vesicles & $\begin{array}{l}\text { Nonregional: Nodes above bifurcation of common iliac arteries, inguinal } \\
\text { nodes, and other distant nodes }\end{array}$ \\
\hline Nodes & (M1b) \\
\hline Bone metastases & \\
\hline - Ascites & \\
\hline Any other finding & \\
\hline
\end{tabular}

Abbreviations: AP, anteroposterior; DRE, Digital rectal examination; DWI, diffusion-weighted imaging; ECE, extracapsular extension; PSA, prostate-specific antigen; PZ, peripheral zone; T2W, T2-weighted; TZ, transition zone.

*Mapping:Identify the index/dominant lesion (one with ECE; if no ECE, the largest one) and map it on the sector map.
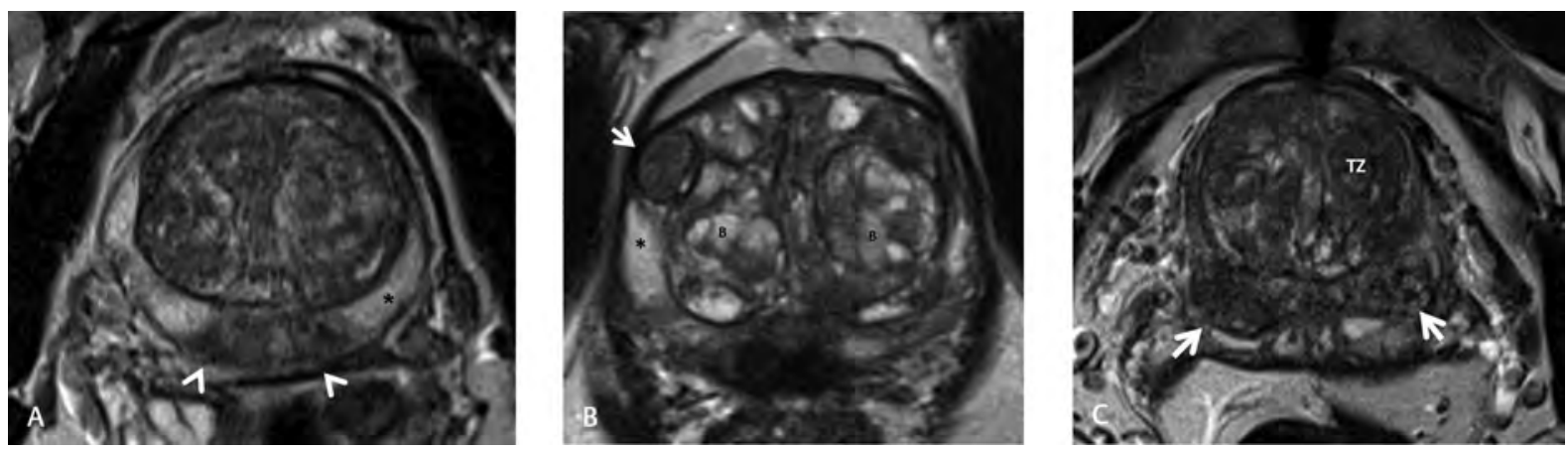

Fig. 16 Pitfalls in prostate MRI. (A) T2 axial image demonstrates symmetric homogenous hypointense lesion in the midline and para-midline peripheral zone at the base (white arrowhead) mimicking a lesion. Rest of the peripheral zone (black asterisk) has normal hyperintense signal. (B) T2 axial image demonstrates benign prostatic hypertrophy of the transition zone with well-defined nodules (B). A well-encapsulated hypointense nodule (white arrow) appears to be in peripheral zone, and actually represents protruding benign nodule from the transition zone. (C) T2 axial image demonstrates hypertrophy of the transition zone (TZ). The peripheral zones at base bilaterally show indistinct, geographical areas of hypointensity (white arrows). Biopsy histology-granulomatous prostatitis.

\section{Funding}

None.

\section{Conflict of Interest \\ None declared.}

\section{References}

1 Bonekamp D, Jacobs MA, El-Khouli R, Stoianovici D, Macura KJ. Advancements in MR imaging of the prostate: from diagnosis to interventions. Radiographics 2011;31(3):677-703

2 Surveillance, Epidemiology and End Results Programme: Cancer Stat Facts: Prostate Cancer. https://seer.cancer.gov/ statfacts/html/prost.html

3 Chen S-L, Wang S-C, Ho C-J, et al. Prostate cancer mortality-to-incidence ratios are associated with cancer care disparities in 35 countries. Sci Rep 2017;7:40003
4 Jain S, Saxena S, Kumar A. Epidemiology of prostate cancer in India. Meta Gene 2014;2:596-605

5 Hariharan K, Padmanabha V. Demography and disease characteristics of prostate cancer in India. Indian $\mathrm{J}$ Urol 2016;32(2):103-108

6 Imaging P. PI-RADS ${ }^{\mathrm{TM}}$ Prostate Imaging-Reporting and Data System. 2015

7 Ploussard G, Epstein JI, Montironi R, et al. The contemporary concept of significant versus insignificant prostate cancer. Eur Urol 2011;60(2):291-303

8 Gordetsky J, Epstein J. Grading of prostatic adenocarcinoma: current state and prognostic implications. Diagn Pathol 2016;11(1):25

9 Chen N, Zhou Q. The evolving Gleason grading system. Chin J Cancer Res 2016;28(1):58-64

10 Bratan F, Niaf E, Melodelima C, et al. Influence of imaging and histological factors on prostate cancer detection and 
localisation on multiparametric MRI: a prospective study. Eur Radiol 2013;23(7):2019-2029

11 Ahmed HU, El-Shater Bosaily A, Brown LC, et al; PROMIS study group. Diagnostic accuracy of multi-parametric MRI and TRUS biopsy in prostate cancer (PROMIS): a paired validating confirmatory study. Lancet 2017;389(10071):815-822

12 Patel P, Mathew MS, Trilisky I, Oto A. Multiparametric MR imaging of the prostate after treatment of prostate cancer. Radiographics 2018;38(2):437-449

13 Coakley FV, Oto A, Alexander LF, et al; Expert Panel on Urologic Imaging. ACR Appropriateness Criteria ${ }^{\circledR}$ Prostate CancerPretreatment Detection, Surveillance, and Staging. J Am Coll Radiol 2017;14(5S):S245-S257

14 Armstrong AJ, Bahnson RR, Victor AD, et al. NCCN Clinical Practice Guidelines in Oncology (NCCN Guidelines): Prostate Cancer. 2016

15 Fulgham PF, Rukstalis DB, Turkbey IB, et al. AUA policy statement on the use of multiparametric magnetic resonance imaging in the diagnosis, staging and management of prostate cancer. J Urol 2017;198(4):832-838

16 Selman SH. The McNeal prostate: a review. Urology 2011;78(6):1224-1228

17 Barentsz JO, Weinreb JC, Verma S, et al. Synopsis of the PI-RADS v2 guidelines for multiparametric prostate magnetic resonance imaging and recommendations for use. Eur Urol 2016;69(1):41-49

18 Hambrock T, Somford DM, Huisman HJ, et al. Relationship between apparent diffusion coefficients at 3.0-T MR imaging and Gleason grade in peripheral zone prostate cancer. Radiology 2011;259(2):453-461

19 Jambor I. Optimization of prostate MRI acquisition and post-processing protocol: a pictorial review with access to acquisition protocols. Acta Radiol Open 2017;6(12): 2058460117745574

20 Rosenkrantz AB, Taneja SS. Radiologist, be aware: ten pitfalls that confound the interpretation of multiparametric prostate MRI. AJR Am J Roentgenol 2014;202(1):109-120
21 Burnside ES, Sickles EA, Bassett LW, et al. The ACR BI-RADS experience: learning from history. J Am Coll Radiol 2009;6(12):851-860

22 Rice TW, Gress DM, Patil DT, Hofstetter WL, Kelsen DP, Blackstone EH. Cancer of the esophagus and esophagogastric junction-major changes in the American Joint Committee on Cancer eighth edition cancer staging manual. CA Cancer J Clin 2017;67(4):304-317

23 Panebianco V, Giganti F, Kitzing YX, et al. An update of pitfalls in prostate mpMRI: a practical approach through the lens of PI-RADS v. 2 guidelines. Insights Imaging 2018;9(1):87-101

24 Schoots IG, Petrides N, Giganti F, et al. Magnetic resonance imaging in active surveillance of prostate cancer: a systematic review. Eur Urol 2015;67(4):627-636

25 Park BH, Jeon HG, Jeong BC, et al. Influence of magnetic resonance imaging in the decision to preserve or resect neurovascular bundles at robotic assisted laparoscopic radical prostatectomy. J Urol 2014;192(1):82-88

26 American Urological Association (AUA) Consensus Statement: Prostate MRI and MRI-targeted biopsy in patients with prior negative biopsy. 2016;(April):1-19

27 Panje C, Panje T, Putora PM, et al. Guidance of treatment decisions in risk-adapted primary radiotherapy for prostate cancer using multiparametric magnetic resonance imaging: a single center experience. Radiat Oncol 2015;10:47

28 Gürses B, Tasdelen N, Yencilek F, et al. Diagnostic utility of DTI in prostate cancer. Eur J Radiol 2011;79(2):172-176

29 Maurer T, Eiber M, Schwaiger M, Gschwend JE. Current use of PSMA-PET in prostate cancer management. Nat Rev Urol 2016;13(4):226-235 